\title{
Alteration in the Plasma Concentrations of Endogenous Organic Anion-Transporting Polypeptide 1B Biomarkers in Patients with Non-Small Cell Lung Cancer Treated with Paclitaxel ${ }^{\mathbb{}}$
}

\author{
Daiki Mori, Hiroo Ishida, Tadahaya Mizuno, Sojiro Kusumoto, Yusuke Kondo, Saki Izumi, \\ Genki Nakata, Yoshitane Nozaki, Kazuya Maeda, Yasutsuna Sasaki, Ken-ichi Fujita, \\ and Hiroyuki Kusuhara
}

Laboratory of Molecular Pharmacokinetics, Graduate School of Pharmaceutical Sciences, the University of Tokyo, Tokyo, Japan (D.M., T.M., Y.K., G.N., K.M., H.K.); Division of Medical Oncology, Department of Medicine (H.I., Y.S.), and Division of Respiratory Medicine and Allergology, Department of Medicine (S.K.), Showa University School of Medicine, Tokyo, Japan; Drug Metabolism and Pharmacokinetics Tsukuba, Tsukuba Research Laboratories, Eisai Co., Ltd., Ibaraki, Japan (S.I., Y.N.); and Division of Cancer Genome and Pharmacotherapy, Department of Clinical Pharmacy, Showa University School of Pharmacy, Tokyo, Japan (K.-i.F.)

Received September 24, 2019; accepted January 28, 2020

\section{ABSTRACT}

Paclitaxel has been considered to cause OATP1B-mediated drugdrug interactions at therapeutic doses; however, its clinical relevance has not been demonstrated. This study aimed to elucidate in vivo inhibition potency of paclitaxel against OATP1B1 and OATP1B3 using endogenous OATP1B biomarkers. Paclitaxel is an inhibitor of OATP1B1 and OATP1B3, with $K_{i}$ of $0.579 \pm 0.107$ and $5.29 \pm 3.87 \mu \mathrm{M}$, respectively. Preincubation potentiated its inhibitory effect on both OATP1B1 and OATP1B3, with $K_{i}$ of $0.154 \pm 0.031$ and $0.624 \pm 0.183 \mu \mathrm{M}$, respectively. Ten patients with non-small cell lung cancer who received $200 \mathrm{mg} / \mathrm{m}^{2}$ of paclitaxel by a 3-hour infusion were recruited. Plasma concentrations of 10 endogenous OATP1B biomarkers-namely, coproporphyrin I, coproporphyrin III, glycochenodeoxycholate-3-sulfate, glycochenodeoxycholate-3glucuronide, glycodeoxycholate-3-sulfate, glycodeoxycholate-3glucuronide, lithocholate-3-sulfate, glycolithocholate-3-sulfate, taurolithocholate-3-sulfate, and chenodeoxycholate-24-glucuronidewere determined in the patients with non-small cell lung cancer on the day before paclitaxel administration and after the end of paclitaxel infusion for 7 hours. Paclitaxel increased the area under the plasma concentration-time curve (AUC) of the endogenous biomarkers 2- to 4-fold, although a few patients did not show any increment in the AUC ratios of lithocholate-3-sulfate, glycolithocholate-3-sulfate, and taurolithocholate-3-sulfate. Therapeutic doses of paclitaxel for the treatment of non-small cell lung cancer $\left(200 \mathrm{mg} / \mathrm{m}^{2}\right)$ will cause significant OATP1B1 inhibition during and at the end of the infusion. This is the first demonstration that endogenous OATP1B biomarkers could serve as surrogate biomarkers in patients.

\section{SIGNIFICANCE STATEMENT}

Endogenous biomarkers can address practical and ethical issues in elucidating transporter-mediated drug-drug interaction (DDI) risks of anticancer drugs clinically. We could elucidate a significant increment of the plasma concentrations of endogenous OATP1B biomarkers after a 3-hour infusion $\left(200 \mathrm{mg} / \mathrm{m}^{2}\right)$ of paclitaxel, a time-dependent inhibitor of OATP1B, in patients with non-small cell lung cancer. The endogenous OATP1B biomarkers are useful to assess the possibility of OATP1Bmediated DDIs in patients and help in appropriately designing a dosing schedule to avoid the DDIs.

\section{Introduction}

Organic anion-transporting polypeptides 1B1/SLCO1B1 (OATP1B1) and $1 \mathrm{~B} 3 / S L C O 1 B 3$ (OATP1B3) are specifically expressed on the

This study was supported by Grants-in-Aid for Scientific Research Foundation B from the Japan Society for the Promotion of Science [17H04100] and Grantsin-Aid for Clinical Pharmacology Research Foundation.

https://doi.org/10.1124/dmd.119.089474.

S This article has supplemental material available at dmd.aspetjournals.org. sinusoidal membrane of hepatocytes to mediate the uptake of their substrates from the blood circulation into hepatocytes for further elimination to the bile and metabolism (Maeda, 2015; Patel et al., 2016; Lee and Ho, 2017; McFeely et al., 2019). OATP1B1 and OATP1B3 are multispecific transporters that recognize various anionic drugs and play key roles in the clearance of such drugs. Therefore, OATP1B-mediated drug-drug interactions (DDIs via induction or inhibition) could modulate the pharmacokinetics of drugs and, thereby, their pharmacological effect or toxicity (Maeda, 2015; Patel et al., 2016;

ABBREVIATIONS: AUC, area under the plasma concentration-time curve; C4, $7 \alpha$-hydroxy-4-cholesten-3-one; CA, cholate; CDCA, chenodeoxycholate; CDCA-24G, chenodeoxycholate-24-glucuronide; CPI, coproporphyrin I; CPIII, coproporphyrin III; DCA, deoxycholate; DDI, drug-drug interaction; E217 $\beta \mathrm{G}$, estradiol 17 $\beta$-D-glucuronide; GCA, glycocholate; GCDCA, glycochenodeoxycholate; GCDCA-G, glycochenodeoxycholate glucuronide; GCDCA-S, glycochenodeoxycholate-3-sulfate; GDCA, glycodeoxycholate; GDCA-G, glycodeoxycholate glucuronide; GDCA-S, glycodeoxycholate sulfate; GLCA-S, glycolithocholate sulfate; HEK293, human embryonic kidney cells 293; KH, Krebs-Henseleit; LCA-S, lithocholate sulfate; NHS, N-Hydroxysulfosuccinimide; OAT, organic anion transporter; OATP, organic anion-transporting polypeptide; TCA, taurocholate; TDCA, taurodeoxycholate; PBPK, physiologically based pharmacokinetic; TLCA-S, taurolithocholate sulfate. 
Lee and Ho, 2017; Asaumi et al., 2019; McFeely et al., 2019). Regulatory agencies in Japan, the United States, and the European Union strongly recommend pharmaceutical industries in their guidelines to assess the OATP1B-mediated DDI risks of their new chemical entities routinely in vitro, and when the predictedrisk is above the regulatory thresholds, to evalute the DDI impact clinically.

The principle of DDI prediction is to compare the unbound concentration at the site of DDI and inhibition constants $\left(\mathrm{K}_{\mathrm{i}}\right)(\mathrm{Giacomini}$ et al., 2010). However, there is a limitation in the current DDI risk prediction for improvement in preclinical stages to avoid false-positive and false-negative predictions (Vaidyanathan et al., 2016). Endogenous biomarkers for drug transporters have emerged as surrogate DDI probes to strengthen the in vitro DDI prediction (Chu et al., 2018; Müller et al., 2018; Rodrigues et al., 2018). Using the typical drug transporter inhibitors, the magnitude of changes of endogenous biomarkers in the area under the plasma concentration-time curves (AUC) and/or renal clearance have been reported for multidrug and toxic compound extrusions, OAT1 and OAT3, and OATP1B1 and OATP1B3. The most important advantage of endogenous biomarkers is that researchers are freed from administration of probe drugs, which could increase the opportunity to assess the DDI risk in humans.

An antimicrotubule agent, paclitaxel, has been used in chemotherapy of various tumors, such as non-small cell lung cancer, breast cancer, and ovarian cancer. The major elimination pathway of paclitaxel from the blood circulation is CYP2C8-mediated metabolism to form $6 \alpha$-hydroxy paclitaxel (Sonnichsen et al., 1995). DDI with paclitaxel as victim drug has been studied in patients who received clopidogrel, which caused time-dependent inhibition of CYP2C8 (Floyd et al., 2012); patients who received both clopidogrel and paclitaxel exhibited higher incidence of severe paclitaxel neuropathy than those treated with paclitaxel and lowdose aspirin, although the impact on paclitaxel pharmacokinetic parameters remains to be determined (Agergaard et al., 2017). In contrast, paclitaxel was also suggested to inhibit OATP1B based on comparison of in vitro $K_{i}$ values for OATP1B1 and OATP1B3 with its clinically relevant unbound concentrations (Marada et al., 2015; Murata et al., 2019). However, the clinical relevance of OATP1B inhibition by paclitaxel has not been confirmed because of ethical and practical issues in conducting clinical DDI studies in healthy subjects or patients. We and other groups identified glycochenodeoxycholate3-sulfate (GCDCA-S) (Takehara et al., 2017, 2018), other sulfated bile acids (Thakare et al., 2017; Mori et al., 2019a; Takehara et al., 2019), coproporphyrin I (CPI) and CPIII (Lai et al., 2016; Barnett et al., 2019), and tetradecanedioate and hexadecanedioate (Yee et al., 2016, 2019; Barnett et al., 2019) as endogenous biomarkers for OATP1B1/1B3. Accumulation of clinical data in healthy subjects to support the suitability of these OATP1B-endogenous biomarkers could allow us to evaluate the OATP1B-mediated DDI risks of anticancer drugs in patients.

This study was a clinical study designed to assess the changes in plasma concentrations of such endogenous OATP1B biomarkers in patients with non-small cell lung cancer who received paclitaxel chemotherapy (paclitaxel $200 \mathrm{mg} / \mathrm{m}^{2}$ given intravenously by a 3-hour infusion plus dexamethasone, diphenhydramine, and famotidine given 30 minutes prior to paclitaxel administration). In addition, the patients also received drug therapy for treatment of their complications (Table 1), but there was no drug that was administered to all patients.

\section{Materials and Methods}

Chemicals. $\left[{ }^{3} \mathrm{H}\right]$ Estradiol $17 \beta$-D-glucuronide $(\mathrm{E} 217 \beta \mathrm{G})$ and $\left[{ }^{3} \mathrm{H}\right]$ cholecystokinin octapeptide were purchased from PerkinElmer Life and Analytical Sciences (Boston, MA). Authentic compounds including stable isotope-labeled compounds

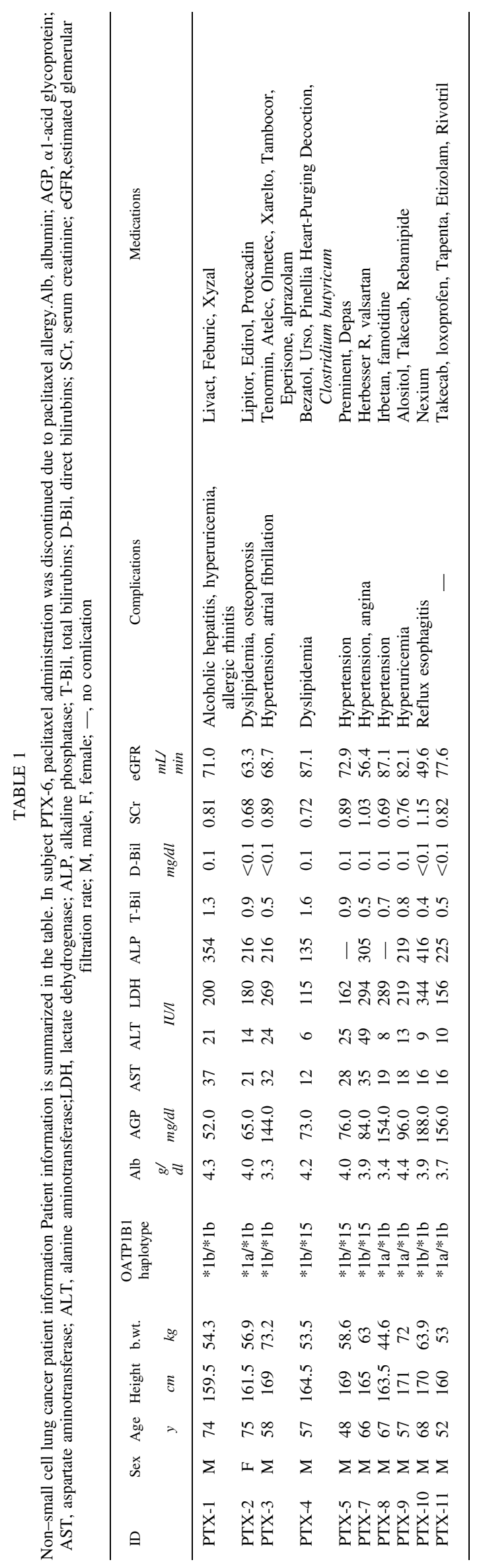


used in this study were all commercially available (Supplemental Materials). Other reagents and organic solvents were of a commercially available analytical grade.

In Vitro Transport Study Using Transporter-Expressing Cells. HEK293 cells, which were stably transfected with OATP1B1, OATP1B3, OAT1, OAT3, or empty vector, were established previously (Deguchi et al., 2004; Hirano et al., 2004). The transport study was performed according to the previous reports (Deguchi et al., 2004; Hirano et al., 2004). Briefly, HEK293 cells were seeded at $2 \times 10^{5}$ cells per well in a 24 -well plate coated with poly-L-lysine $(50 \mathrm{mg} / \mathrm{l})$ and poly-L-ornithine $(50 \mathrm{mg} / \mathrm{l})$. After 48 hours, the medium was substituted by Dulbecco's modified Eagle's medium containing $5 \mathrm{mM}$ sodium butyrate. After 24 hours, the transport study was conducted. Cells were washed twice and preincubated with Krebs-Henseleit $(\mathrm{KH})$ buffer at $37^{\circ} \mathrm{C}$ for 5 minutes. Then, substrates were added to initiate the uptake. At the designated times, incubation buffer was removed, and ice-cold $\mathrm{KH}$ buffer was added to terminate the uptake.

To evaluate the inhibition potency of paclitaxel and $6 \alpha$-hydroxy paclitaxel, either paclitaxel or $6 \alpha$-hydroxy paclitaxel was added simultaneously with the test substrate. When the time dependence of the inhibitory effect was assessed (shown in Fig. 1 and Supplemental Fig. 8), cells were preincubated in the presence of paclitaxel or $6 \alpha$-hydroxy paclitaxel for 15 minutes. In a separate experiment (shown in Supplemental Fig. 6A), cells were preincubated in $\mathrm{KH}$ buffer containing paclitaxel for the designated periods (5, 10, 30, 60 minutes) and then washed. After washing, uptake was initiated in the absence of inhibitor. To assess the duration of the inhibitory effect (shown in Supplemental Fig. 6B), cells were preincubated with $\mathrm{KH}$ buffer containing paclitaxel for 30 minutes and incubated with paclitaxel-free $\mathrm{KH}$ buffer for the designated periods $(0,10,30,60$ minutes). Then, uptake was initiated in the absence of paclitaxel.

For quantification of nonradiolabeled compounds, the cells were collected in Milli-Q water using a scraper and disrupted using a bioruptor (Sonic-bio, Kanagawa, Japan). A $20-\mu 1$ aliquot was mixed with $60 \mu l$ of acetonitrile containing the corresponding internal standard solution. After vortex mixing, the mixtures were centrifuged $\left(20,000 \mathrm{~g}\right.$ for 5 minutes at $\left.4^{\circ} \mathrm{C}\right)$, and the supernatants were used for the following liquid chromatography-tandem mass spectrometry analyses. For quantification of the radioactivity, the cells were solubilized with $200 \mu 10.2 \mathrm{~N} \mathrm{NaOH}$ overnight at $4^{\circ} \mathrm{C}$ and then neutralized with $100 \mu 10.4 \mathrm{~N} \mathrm{HCl}$. Aliquots of the lysates $(200 \mu \mathrm{l})$ were transferred to scintillation vials containing a scintillation cocktail (Clear-sol I; Nacalai Tesque, Kyoto, Japan), and the radioactivity was measured in a liquid scintillation counter (LS6000SE; Beckman Instruments, Inc., Fullerton, CA). The protein concentration was determined using the protein assay method of Lowry et al. (1951), using bovine serum albumin as the protein standard.

Western Blotting to Detect OATP1B1 and OATP1B3 in Whole Cells and Cell Surface. Cells were harvested by cell scraper and collected by centrifugation $\left(12,000 \mathrm{~g}, 5\right.$ minutes, $\left.4^{\circ} \mathrm{C}\right)$. They were lysed by cell lysis buffer (Cell Signaling, Danvers, MA). The samples were centrifuged at $12,000 \mathrm{~g}$ for 10 minutes at $4^{\circ} \mathrm{C}$, and then the supernatants were used in the analysis. The $4 \times$ SDS sample buffer (12\% SDS, 25\% glycerol, $150 \mathrm{mM}$ Tris- $\mathrm{HCl} \mathrm{pH} 7.0,0.05 \%$ bromophenol blue, $6 \% \beta$-mercaptoethanol) and 2-mercaptoethanol were added $1 / 4$ and $1 / 30$ volume of the sample, respectively, and heated at $60^{\circ} \mathrm{C}$ for 5 minutes. Then, the whole-cell samples were subjected to Western blot analysis.

For cell surface analysis of OATP1B1 and OATP1B3 proteins, cell surface biotinylation was conducted as previously described (Mizuno et al., 2015). Briefly, cells were incubated with $\mathrm{N}$-Hydroxysulfosuccinimide (NHS)-SS-Biotin solution $(0.5 \mathrm{mg} / \mathrm{ml}$; Pierce Biotechnology, Inc., Rockford, IL) for 20 minutes at $4{ }^{\circ} \mathrm{C}$ and washed using PBS supplemented with $\mathrm{Ca}^{2+}, \mathrm{Mg}^{2+}$, and $100 \mathrm{mM}$ glycine before preparation of cell lysate with cell lysis buffer (Cell Signaling). The cell lysate was mixed with $50 \mu \mathrm{l}$ of streptavidin-agarose (Thermo Fisher Scientific, Waltham, MA) and kept under end-over-end rotation for 2 hours at $4^{\circ} \mathrm{C}$. After centrifugation at $5900 \mathrm{~g}$ for 1 minute at $4^{\circ} \mathrm{C}$, the supernatant was removed. The beads were washed with lysis buffer ( $50 \mathrm{mM}$ Tris, $150 \mathrm{mM} \mathrm{NaCl}, 5 \mathrm{mM}$ EDTA, $1 \%$ Triton $\mathrm{X}-100, \mathrm{pH} 7.5)$ twice, high-salt buffer $(50 \mathrm{mM}$ Tris, $500 \mathrm{mM} \mathrm{NaCl}$, 5 mM EDTA, $0.1 \%$ Triton X-100, pH 7.5) twice, and low-salt buffer (50 mM Tris, $\mathrm{pH} 7.5$ ) once. Biotinylated proteins were eluted with $40 \mu \mathrm{l}$ of loading buffer (Milli-Q water/4× SDS loading buffer/2-mercaptoethanol, 35/4/1) for 1 hour at $60^{\circ} \mathrm{C}$, and obtained specimens were subjected to Western blotting analysis.

The specimens were loaded into wells of a 10\% SDS-PAGE plate with a $3.75 \%$ stacking gel and subjected to immunoblotting as previously described (Hirano et al., 2004). Immunoreactivity was detected with an ECL Prime Western Blotting Detection Kit (Amersham Biosciences, Piscataway, NJ).
Clinical Study Design. This was a prospective study performed with Japanese patients with non-small cell lung cancer who received the first cycle of paclitaxel and carboplatin regimen. To evaluate the difference in plasma concentration profiles of the endogenous substrates with or without the paclitaxel infusion, the blood samples were collected on two consecutive days at the same time points. On day 1, plasma samples were collected before and after the paclitaxel infusion as shown below, whereas on day 0 , plasma samples were collected at the same time points as day 1 . The study was conducted in accordance with the principles of the Declaration of Helsinki. This study protocol was approved by the Institutional Review Board of Showa University and Graduate School of Pharmaceutical Sciences, The University of Tokyo. All patients were asked for written informed consent for their peripheral blood samples and medical information to be used for research purposes. The study was registered at the University Hospital Medical Information Network-Clinical Trials Registry Japan (UMIN000032370).

Eligibility. All patients 20 years or older with metastatic or recurrent and histologically confirmed non-small cell lung cancer who received paclitaxel had an Eastern Cooperative Oncology Group performance status of 0 to 1 and no history of chemotherapy within 4 weeks were eligible. Each patient was confirmed to have adequate bone marrow function (absolute neutrophil count, at least $1.5 \times 10^{9} / \mathrm{l}$; platelet count at least $150 \times 10^{9} / 1$ ), liver function (serum bilirubin level less than $1.5 \mathrm{mg} / \mathrm{dl}$; transaminases less than 2.5 times the upper limit of normal), and renal function (serum creatinine level less than $1.5 \mathrm{mg} / \mathrm{dl}$ ) (Table 1).

Treatment. The therapeutic dose of paclitaxel $\left(200 \mathrm{mg} / \mathrm{m}^{2}\right)$ and carboplatin (area under the blood concentration-time curve of $6 \mathrm{mg} / \mathrm{ml} \times \mathrm{min}$ ) was administered to patients with non-small cell lung cancer. Combination chemotherapy with bevacizumab $(15 \mathrm{mg} / \mathrm{kg})$ was permitted. Paclitaxel was administered as an intravenous drip infusion over the course of 3 hours after the patient received premedication with oral $50 \mathrm{mg}$ of diphenhydramine followed by intravenously given $3 \mathrm{mg}$ of granisetron, $50 \mathrm{mg}$ of ranitidine, and $20 \mathrm{mg}$ of dexamethasone over the course of 10 minutes (Supplemental Fig. 1). Thereafter, carboplatin was given as an intravenous drip infusion over the course of 1 hour. The patients were given a dietary supplement containing an equivalent amount of total daily lipids to minimize the influence of these dietary ingredients on the synthesis or disposition of the endogenous substrates of OATP1B1 and OATP1B3, such as bile acids. Note that regular meals were served to patients at 8:00 AM and 12:00 PM with the same menu on day 0 and day 1, and paclitaxel infusion was performed from 11:00 AM to 2:00 PM.

Blood Sampling. Blood samples were taken from the arm opposite the infusion site at the beginning of paclitaxel infusion and 0,2, 4, and 7 hours after the end of the 3-hour infusion of paclitaxel on day 1. Blood samples were also collected at the same time points on day 0 to assess the difference in plasma concentration profiles of the endogenous substrates in the presence or absence of paclitaxel infusion. The blood samples were immediately centrifuged, and the plasma was stored at $-80^{\circ} \mathrm{C}$ until analysis.

Genotyping of SLCO1B1 in Patients. This study focused on two SLCO1B1 single nucleotide polymorphisms, $388 \mathrm{G}>\mathrm{A}$ and $521 \mathrm{~T}>\mathrm{C}$, that form haplotypes $* 1 \mathrm{a}$, $* 1 \mathrm{~b}$, and $* 15$. The genotypes were determined as described previously (Akiyama et al., 2012) using genomes prepared from peripheral blood cells of the patients.

Determination of the Unbound Fraction of Paclitaxel in the Plasma. The unbound fraction of paclitaxel in the plasma $\left(f_{p}\right)$ was determined at 0 and 7 hours using a High Throughput Dialysis Model HTD96b and Dialysis Membrane Strips MWCO (12-14 kDa) obtained from HTDialysis, LLC (Gales Ferry, CT).

Quantification of Paclitaxel and 6 $\alpha$-Hydroxy Paclitaxel in the Plasma Specimens. A plasma aliquot $(20 \mu \mathrm{l})$ was added to acetonitrile $(180 \mu \mathrm{l})$, vortexed, and centrifuged at $20,000 \mathrm{~g}$ for 5 minutes for protein precipitation. The supernatant $(10 \mu \mathrm{l})$ was mixed with $0.1 \%$ formic acid/acetonitrile $=1 / 1$ solvents $(150 \mu \mathrm{l})$ and analyzed using liquid chromatography-tandem mass spectrometry. Chromatography was performed on a Prominence Ultra Fast Liquid Chromatography system (Shimadzu, Kyoto, Japan), and separation was achieved as described in Supplemental Methods. Data were collected on an AB Sciex API5500 (QTRAP) mass spectrometer (Foster City, CA) using positive Turbo IonSpray electrospray ionization and Multiple Reaction Monitoring mode (Supplemental Methods). Data acquisition and processing were carried out with Analyst software version 1.6.2. (Applied Biosystems/MDS Sciex, Foster City, Canada).

Quantification of Endogenous OATP1B Biomarkers and Bile Acids in the Plasma Specimens. A QTRAP5500 mass spectrometer (AB Sciex) equipped with Prominence Ultra Fast Liquic Chromatography. system (Shimadzu) or Nexera Ultra High Performance Liquid Chromatography (Shimadzu) was used 
OATP1B1

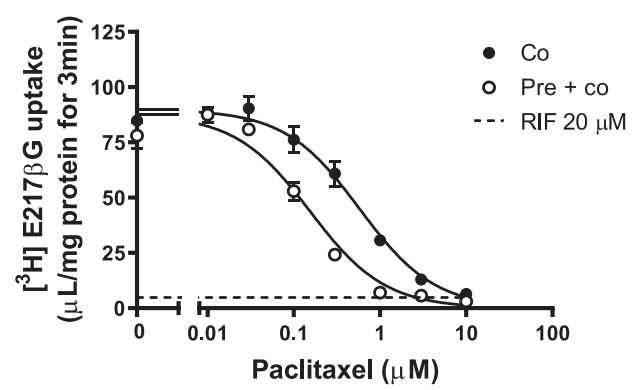

OATP1B3

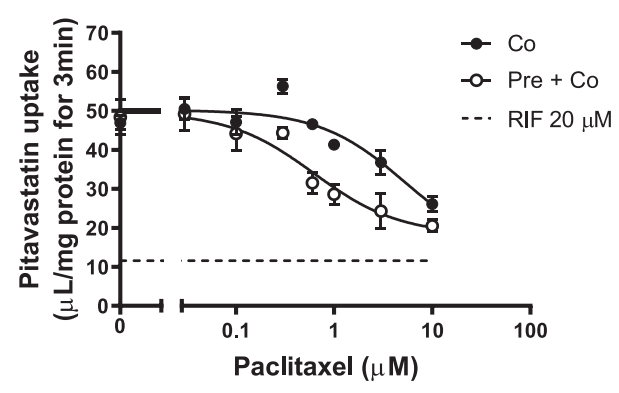

Fig. 1. Inhibitory effect of paclitaxel on the OATP1B1- and OATP1B3-mediated uptake in HEK293 cells. The uptake of $\left[{ }^{3} \mathrm{H}\right] \mathrm{E} 217 \beta \mathrm{G}$ by HEK293 cells stably expressing OATP1B1 and that of pitavastatin $(0.3 \mu \mathrm{M})$ by HEK293 cells stably expressing OATP1B3 for 3 minutes with $(\bigcirc)$ or without $(\mathbf{O})$ paclitaxel preincubation $(15$ minutes) was determined in the absence and presence of paclitaxel at the designated concentrations or rifampicin $(20 \mu \mathrm{M})$. The solid line represents the fitted line obtained by nonlinear regression analysis as described in the Materials and Methods section. The dotted line represents the mean value of the uptake of $\left[{ }^{3} \mathrm{H}\right] \mathrm{E} 217 \beta \mathrm{G}$ in the presence of rifampicin. Each symbol represents the mean value with S.E.M. $(n=3)$.Co, coincubation; Pre $+\mathrm{Co}$, preincubation plust coincubation; RIF, rifampicin. for quantification of endogenous compounds. Quantification of C4, bile acids, and bile acid sulfates (LCA-S, TLCA-S, and GLCA-S) was performed as described previously (Mori et al., 2019a,b). The measurement method is summarized in Supplemental Methods. For separation of stereoisomers, such as CPI and CPIII, GCDCA-S and GDCA-S, and GCDCA-G and GDCA-G, the chromatographic separation was modified as described in the Supplemental Methods.Supplemental Methods

Calculation of AUC of the Endogenous Compounds. The AUC was calculated from -3 to 10 hours using the linear trapezoidal method using Excel.

Determination of the Inhibition Constants $\left(K_{i}\right)$ of Paclitaxel and $6 \alpha$-Hydroxy Paclitaxel for OATP1B1 and OATP1B3. Iterative nonlinear least-squares method using GraphPad Prism8 (GraphPad Software, San Diego, CA) was conducted to determine the $K_{i}$ values of paclitaxel or $6 \alpha$-hydroxy paclitaxel. The uptake of test compound determined in the presence and absence of inhibitor [ $\mathrm{CL}_{\text {uptake (+inhibitor) }}$ and $\mathrm{CL}_{\text {uptake (control), }}$ respectively] was fitted to the following equation:

$$
C L_{\text {uptake }(+ \text { inhibitor })}=\frac{C L_{\text {uptake }(\text { control })}}{1+I / K_{i}}
$$

where I represents the inhibitor concentration.

Statistics. Paired $t$ test was used to examine statistical significance in the effect of paclitaxel administration on the plasma concentrations of endogenous OATP1B biomarkers using GraphPad Prism8 (GraphPad Software). Geometric means and $95 \%$ confidence of intervals of the AUC ratio were calculated using GraphPad Prism8.

\section{Results}

Effect of Paclitaxel on OATP1B1- and OATP1B3-Mediated Uptake in HEK293 Cells. Paclitaxel inhibited the OATP1B1-mediated uptake of $\left[{ }^{3} \mathrm{H}\right] \mathrm{E} 217 \beta \mathrm{G}$ in a concentration-dependent manner (Fig. 1), with $\mathrm{K}_{\mathrm{i}}$ of $0.579 \pm 0.107 \mu \mathrm{M}$ with a competitive manner (Supplemental Fig. 2). A 15-minute preincubation with paclitaxel potentiated its inhibitory effect, reducing the $\mathrm{K}_{\mathrm{i}}$ to $0.154 \pm 0.031 \mu \mathrm{M}$ (Fig. 1). Paclitaxel showed lower inhibition potency to OATP1B3 than OATP1B1 (Fig. 1), with the estimated $\mathrm{K}_{\mathrm{i}}$ of $0.624 \pm 0.183$ and 5.29 $\pm 3.87 \mu \mathrm{M}$ with or without preincubation, respectively (Fig. 1). OATP1B1 and OATP1B3 were not inhibited by drugs administered prior to paclitaxel administration (Supplemental Fig. 3). Paclitaxel did not inhibit either OAT1 or OAT3 at $10 \mu \mathrm{M}$ (Supplemental Fig. 4).

Plasma Concentration-Time Profile of Paclitaxel in Patients with Non-Small Cell Lung Cancer. Plasma concentrations of paclitaxel and its hydroxy metabolite, $6 \alpha$-hydroxy paclitaxel, were determined in the patients (Fig. 2). Plasma concentrations of $6 \alpha$-hydroxy paclitaxel accounted for $1 / 10$ of those of paclitaxel (Fig. 2). The $f_{p}$ of paclitaxel at 0 and 7 hours was determined in all of the subjects. The mean values were $0.0158 \pm 0.0042$ and $0.0354 \pm 0.0247$, respectively, which were similar to the previously reported values $(0.036-0.079)$ (Brouwer et al., 2000). The unbound concentration of paclitaxel was calculated using individual $f_{p}$ and $C_{\max }$ at 0 hours. It was somewhat below the $K_{i}$ for OATP1B1 with preincubation (Supplemental Fig. 5).

Effect of Paclitaxel Administration on the Plasma Concentrations of the Endogenous OATP1B Biomarkers. Plasma concentrations of 10 endogenous biomarkers, three porphyrin metabolites (CPI and CPIII), and bile acid glucuronides or sulfate (GCDCA-S, GCDCA-G, GDCA-S, GDCA-G, LCA-S, GLCA-S, TLCA-S, CDCA-24G) were determined on day 0 (baseline) and day 1 (before and after paclitaxel administration) (Fig. 3). During the observed time ( -3 to 7 hours), there was no obvious change in the plasma concentrations on day 0 . After a 3-hour infusion of paclitaxel, the plasma concentrations of the endogenous biomarkers were higher than the predose level or baseline (day 0). The time to reach maximum concentration differed among the biomarkers: end of infusion, CPI, CPIII, and CDCA-24G; 0-2 hours postinfusion, GCDCA-S, GDCA-S, LCA-S, GLCA-S, and TLCA-S; 4 hours postinfusion, GCDCA-G and GDCA-G. The increased plasma concentrations did not return to the corresponding baseline levels by 7 hours postinfusion. Note that LCA-S, GLCA-S, and TLCA-S could not be quantified in one patient (PTX-5).

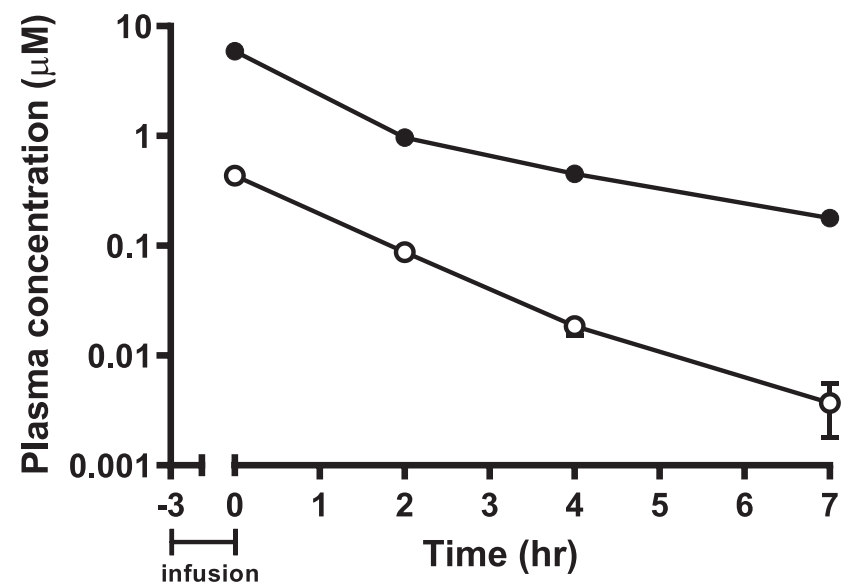

Fig. 2. Plasma concentrations of paclitaxel and $6 \alpha$-hydroxy paclitaxel. Plasma concentrations of paclitaxel (closed symbols) and $6 \alpha$-hydroxy paclitaxel (open symbols) were determined at designated time points in patients with non-small cell lung cancer after a 3-hour intravenous infusion of paclitaxel $\left(200 \mathrm{mg} / \mathrm{m}^{2}\right)$ from -3 to 0 hours. Each symbol represents the mean value, and error bars representing S.E.M. $(n=10)$ were within the symbol. 

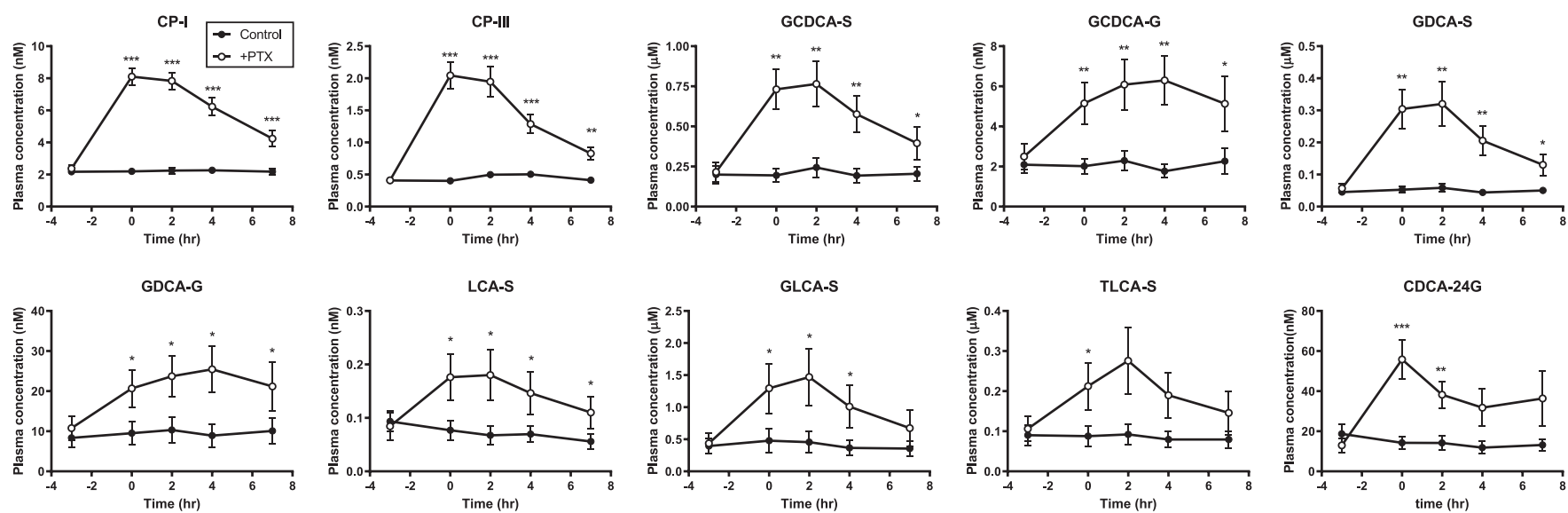

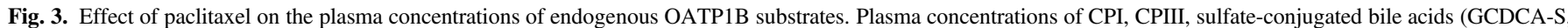

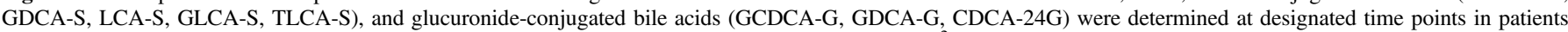

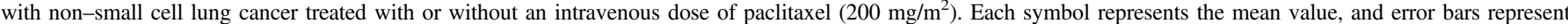
S.E.M. $(n=10)$. ${ }^{*} P<0.05 ; * * P<0.01 ; * * * P<0.001$.

The interindividual difference in the response to paclitaxel administration is shown as AUC in Fig. 4. The mean and range of the AUC ratio are shown in Table 2. There were nonresponders (AUC ratio $<1.25$ ) to paclitaxel for LCA-S, GLCA-S, and TLCA-S; LCA-S, PTX-7, and PTX-10; GLCA-S, PTX-7, and PTX-9; and TLCA-S, PTX-7, PTX-8, and PTX-9. Because of these nonresponders, the mean ratio of AUC was somewhat smaller for these metabolites. The mean ratio of AUC was similar among the other metabolites (Table 2).

Effect of Paclitaxel Administration on the Plasma Concentrations of the Intermediate Metabolites of Bile Acid Synthesis and Bile Acids. The plasma concentrations of $\mathrm{C} 4$ and bile acids were also measured in the patients with or without paclitaxel administration. The plasma concentrations of $\mathrm{C} 4$ on day 0 were similar throughout the study, whereas those on day 1 decreased significantly over time (Fig. 5A). Plasma concentrations of bile acids, including the precursor of the glucuronide and sulfate conjugates, such as GCDCA and GDCA, were also determined. The mean values increased at the end of infusion for TCA, GCA, GCDCA, GDCA, and TDCA, followed by a rapid decline to the baseline levels, whereas there was no difference in the plasma concentrations of CDCA and DCA with and without paclitaxel administration (Fig. 5A). There was a clear interindividual difference in the
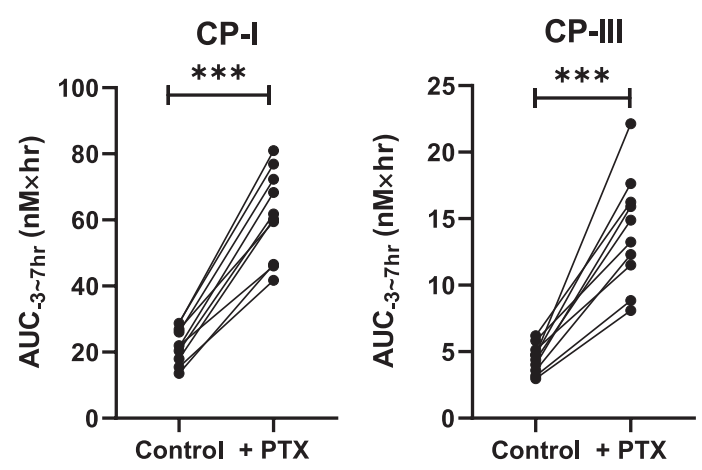

GDCA-G

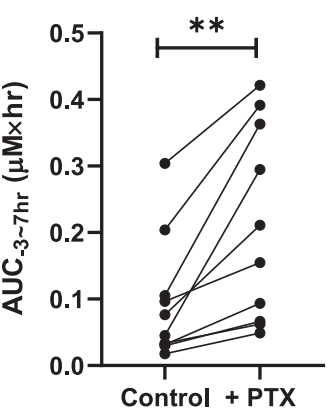

LCA-S

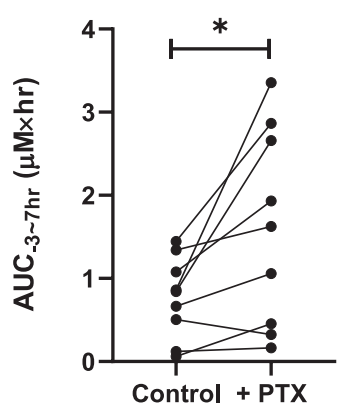

GCDCA-S

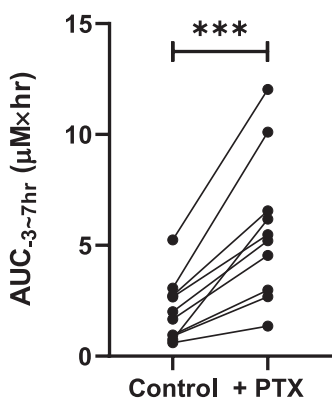

GLCA-S

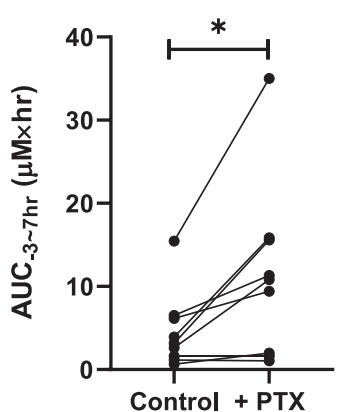

GCDCA-G

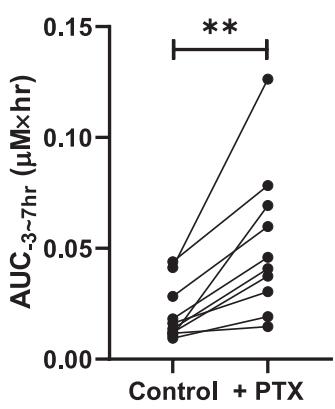

TLCA-S

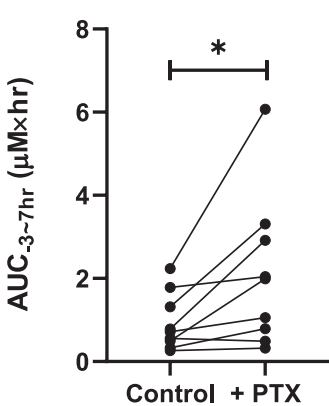

GDCA-S
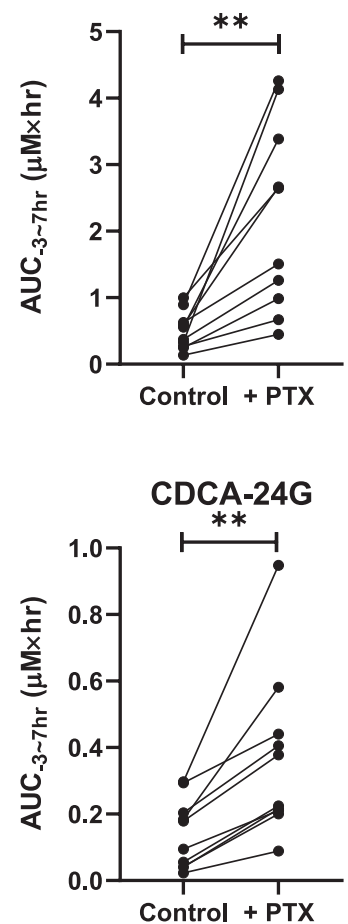

Fig. 4. Area under the plasma concentration-time curves for endogenous OATP1B substrates with and without paclitaxel administration. The symbols represent AUC values in 10 patients calculated using the linear trapezoidal rule from time -3 to 7 hours. $* P<0.05 ; * * P<0.01 ; * * * P<0.001$. 
TABLE 2

Effect of paclitaxel on $\mathrm{AUC}_{-3-7 \mathrm{~h}}$ of endogenous OATP1B substrates $\mathrm{AUC}_{-3-7 \mathrm{~h}}$ of the endogenous OATP1B substrates were calculated. $\mathrm{AUC}_{-3-7 \mathrm{r}}$ are presented as mean \pm S.E.M. Fold changes are presented as GMR and $90 \% \mathrm{CI}(\mathrm{n}=10)$. GMR, geometric mean ratio.

\begin{tabular}{lcccr}
\hline Compounds & & Control & + Paclitaxel & Fold Change \\
\hline CPI & $(\mathrm{nM} \times \mathrm{h})$ & $22.1 \pm 1.7$ & $61.4 \pm 4.3$ & $2.8(2.5,3.1)$ \\
CPIII & $(\mathrm{nM} \times \mathrm{h})$ & $4.48 \pm 0.34$ & $14.1 \pm 1.3$ & $3.1(2.6,3.7)$ \\
GCDCA-S & $(\mu \mathrm{M} \times \mathrm{h})$ & $2.06 \pm 0.45$ & $5.71 \pm 1.0$ & $2.9(2.2,3.9)$ \\
GDCA-S & $(\mu \mathrm{M} \times \mathrm{h})$ & $0.501 \pm 0.09$ & $2.2 \pm 0.45$ & $4.0(2.8,5.8)$ \\
LCA-S & $(\mu \mathrm{M} \times \mathrm{h})$ & $0.694 \pm 0.16$ & $1.46 \pm 0.38$ & $2.0(1.2,3.5)$ \\
GLCA-S & $(\mu \mathrm{M} \times \mathrm{h})$ & $4.15 \pm 1.4$ & $10.4 \pm 3.3$ & $2.2(1.4,3.6)$ \\
TLCA-S & $(\mu \mathrm{M} \times \mathrm{h})$ & $0.857 \pm 0.22$ & $1.94 \pm 0.57$ & $2.0(1.3,3.0)$ \\
GCDCA-G & $(\mathrm{nM} \times \mathrm{h})$ & $20.6 \pm 4.1$ & $52.2 \pm 10$ & $2.5(1.8,3.4)$ \\
GDCA-G & $(\mathrm{nM} \times \mathrm{h})$ & $94.4 \pm 29$ & $211 \pm 46$ & $2.5(1.8,3.4)$ \\
CDCA-24G & $(\mathrm{nM} \times \mathrm{h})$ & $141 \pm 33$ & $369 \pm 79$ & $3.0(2.2,4.0)$ \\
& & & & \\
\hline
\end{tabular}

baseline levels of bile acids and effect of paclitaxel administration on AUC of bile acids (Fig. 5B).

\section{Discussion}

DDI risk assessment of anticancer drugs remains mainly limited to in vitro or animal data because elucidating the clinical relevance of the inhibition at their therapeutic dose ranges is ethically and practically difficult. Paclitaxel is one of the anticancer drugs that were suggested to inhibit OATP1B by conventional prediction methods (Marada et al., 2015; Murata et al., 2019). Taking advantage of endogenous biomarkers, circumventing the need for investigators to administer probe drugs, we assessed OATP1B1 and OATP1B3 inhibition potency of paclitaxel at its therapeutic dose for the treatment of non-small cell lung cancer.

Consistent with previous reports, the in vitro inhibition study showed the concentration-dependent inhibition of OATP1B1 and OATP1B3 by paclitaxel (Fig. 1). Competitive inhibition was confirmed for OATP1B1 inhibition (Supplemental Fig. 2), whereas absence of paclitaxel effect on renal organic anion transporters, OAT1 and OAT3 (Supplemental Fig. 4), excludes nonspecific effects, at least during the experiments. Furthermore, the inhibition of OATP1B1 and OATP1B3 by paclitaxel was time-dependent (Fig. 1; Supplemental Fig. 6), which had been reported for cyclosporin A (Shitara and Sugiyama, 2017; Tátrai et al., 2019). A metabolite of paclitaxel, $6 \alpha$-hydroxy paclitaxel, retained a time-dependent effect on OATP1B1 with similar inhibition potency, whereas it no longer showed time-dependent inhibition of OATP1B3 (Supplemental Fig. 8). The time dependence of OATP1B1 inhibition appears to be reversible because the activity partially recovered after incubation in the absence of paclitaxel (Supplemental Fig. 6). The possibility that paclitaxel treatment decreased the expression of OATP1B1 and OATP1B3 proteins in whole cells and on the cell surface was excluded (Supplemental Fig. 7). Shitara and Sugiyama
A
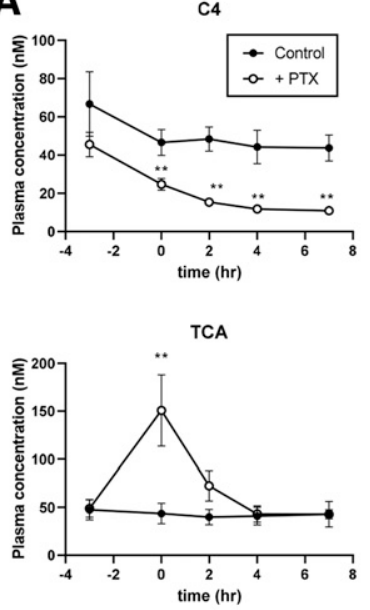

DCA

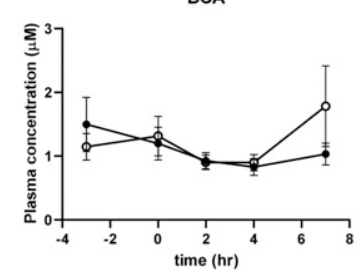

CA

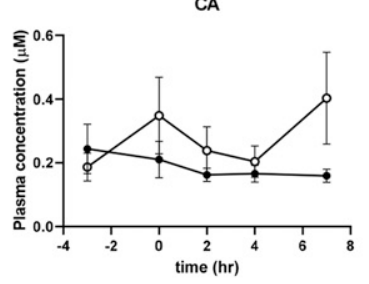

CDCA

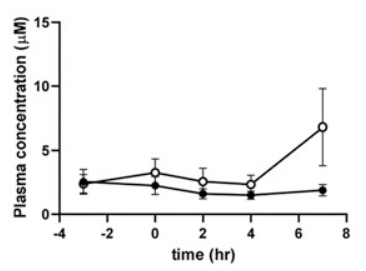

GDCA

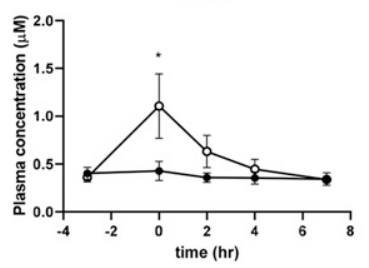

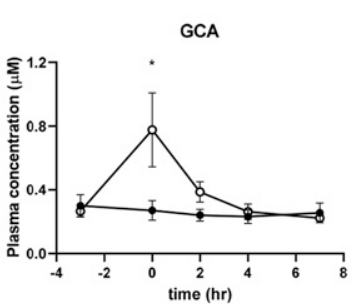

GCDCA

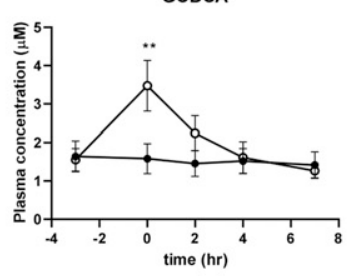

TDCA

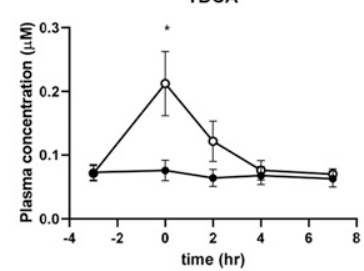

B
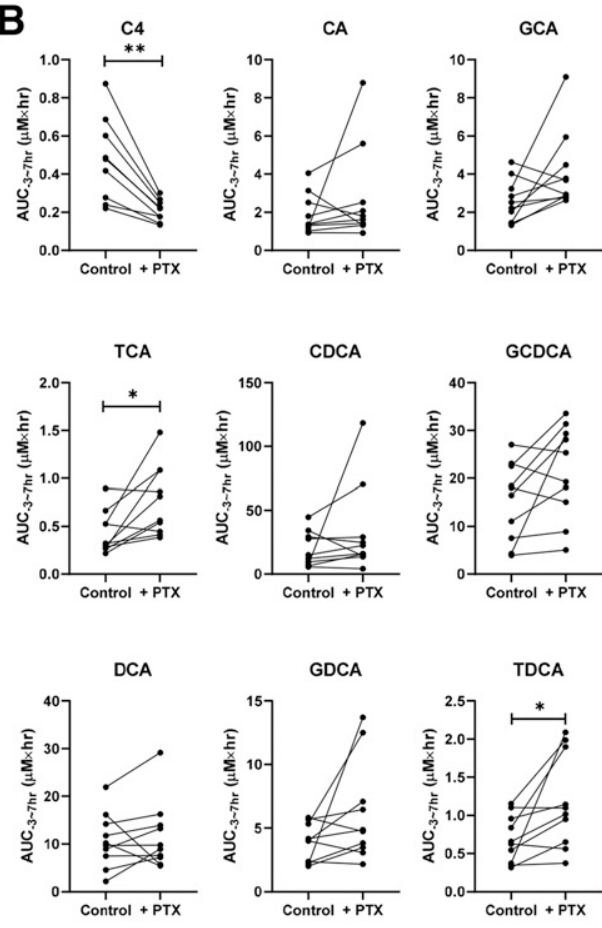

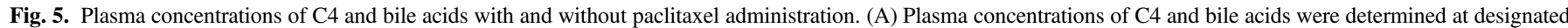

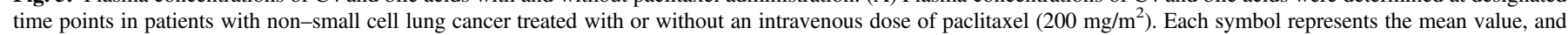

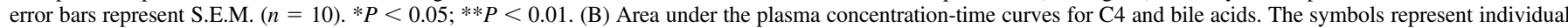
values of AUC calculated using the trapezoidal rule from time -3 to 7 hours. ${ }^{*} P<0.05 ; * * P<0.01$. 
(2017) proposed a trans-inhibition model to account for the timedependent effect of cyclosporin A (Shitara and Sugiyama, 2017). Paclitaxel may also have an inhibitory effect on OATP1B1 in the plasma membrane or from the cytosolic compartment, where paclitaxel accumulated during incubation. It is also possible that paclitaxel inhibits the maintenance of the driving force for OATP1B1 and OATP1B3, which has not been identified, or post-translational modifications such as phosphorylation, as has been proposed for other drug transporters (Sprowl et al., 2016). It should be noted that, as pointed out for OATP1B1 (Izumi et al., 2015), the inhibitory effect of paclitaxel for OATP1B3 was substrate-dependent; the inhibitory effect of paclitaxel on OATP1B3-mediated $\left[{ }^{3} \mathrm{H}\right]$ cholecystokinin octapeptide uptake was weaker than that on pitavastatin uptake (Supplemental Fig. 9). Careful selection of test OATP1B3 probe is required for conservative DDI risk assessment.

Comparison of the unbound concentration of paclitaxel in the plasma, estimated as the product of $\mathrm{f}_{\mathrm{p}}$ and $\mathrm{C}_{\max }$, with its $\mathrm{K}_{\mathrm{i}}$ for OATP1B1 and OATP1B3 (Supplemental Fig. 5), suggests that at this dose regimen, paclitaxel inhibits OATP1B1 more potently than OATP1B3. The plasma concentrations of the endogenous OATP1B biomarkers were significantly higher at the end of paclitaxel infusion than the two control values; baselines were determined on the day before paclitaxel administration, and values were determined before starting the administration of paclitaxel (confirming the interday difference) (Fig. 3). The magnitude of changes in CPI concentrations induced by paclitaxel administration was similar to that previously observed in the subjects with SLCO1B1 *15 homozygotes (Mori et al., 2019a) and those who received $300 \mathrm{mg}$ of rifampicin (Takehara et al., 2018). Previously, the AUC ratio of GCDCA-S (4.3) when $300 \mathrm{mg}$ of rifampicin was administeredwas greater than that of CPI (2.3) (Takehara et al., 2018), whereas the AUC ratios of CPI and GCDCA-S were similar in this study (Table 2). Such a difference may arise from the in vivo inhibition potency of OATP1B1 and OATP1B3 between rifampicin and paclitaxel or disease-related factors. The AUC ratio of LCA-S, GLCA-S, and TLCA-S did not change in two to three patients in this study. Such nonresponse of sulfate conjugates of secondary bile acids to OATP1B inhibition was also observed in the rifampicin study (Mori et al., 2019b). The reason for this remains unknown, but using such metabolites as surrogate OATP1B probes should be avoided.

The plasma concentrations of bile acids were also determined on both days. Those on day 1 at some time points were higher than the predose levels (Fig. 5; Supplemental Table 1). One patient who showed a higher AUC ratio of GCDCA (6.8) than others (0.8-1.7) also showed the highest AUC ratio of GCDCA-S (13) and GCDCA-G (6.4) compared with others (2.4-6 and 1.2-3.1, respectively), but the AUC ratio of CPI was similar. Therefore, the AUC ratio of GCDCA-S and GCDCA-G might be influenced not only by OATP1B inhibition but also by the interday variation or effect of paclitaxel and/or the concomitantly administered drug on GCDCA. The possibility that paclitaxel inhibits sodium-taurocholate co-transporting polyppetide can be excluded because of absence of the effect on sodium-taurocholate cotransporting polyppetide-mediated uptake of $\left[{ }^{3} \mathrm{H}\right]$ taurocholate at $10 \mu \mathrm{M}$ (data not shown). In addition, $\mathrm{C} 4$, an intermediate metabolite of bile acid synthesis from cholesterol, continued to decline on day 1 after paclitaxel administration (Fig. 5). Paclitaxel therapy might affect bile acid synthesis.

Although limited in patient number, effect of SLCO1B1 genotypes (wild-type homozygotes and $* 15$ heterozygotes) and biochemical test results were shown in Supplemental Figs. 10 and 11. A nonsynonymous single nucleotide polymorphism of SLCO1B1 (c. $521 \mathrm{~T}>\mathrm{C}$, p.Val174Ala) is well-known to be associated with higher systemic exposure of OATP1B1 substrate drugs (Lee and Ho, 2017; Yee et al.,
2018). In Japanese people, this mutation is associated in a haplotype $(* 15)$ with another nonsynonymous mutation (c. 388A $>$ G, p.Asn130Asp) (Nishizato et al., 2003). Japanese individuals homozygous for the $* 15$ allele showed higher plasma concentrations of CPI and GCDCA-S than homozygotes of reference types and heterozygotes (Mori et al., 2019a). Consistent with our previous report, individuals heterozygous for this mutation did not show any difference in the plasma concentrations of these substrates (Supplemental Fig. 10), whereas the AUC ratio of CPIII, but not CPI, appeared to be SLCO1B1 genotype-dependent (Supplemental Fig. 10), which requires further confirmation in future studies. There was no significant effect of biochemical test results on the AUC ratio of CPI (Supplemental Fig. 11) and other test compounds (data not shown).

Together with previously reported practices (Lai et al., 2016; Yee et al., 2016; Shen et al., 2017; Takehara et al., 2017, 2018; King-Ahmad et al., 2018; Kunze et al., 2018; Liu et al., 2018; Müller et al., 2018; Cheung et al., 2019), this study provides more convincing evidence to expand application of the endogenous OATP1B biomarkers to assess in vivo OATP1B inhibition under conditions in which administration of probe drugs is limited or under those closer to actual clinical practice. In addition to paclitaxel, mitoxantrone and etoposide were suggested to cause significant inhibition of OATP1B3 (Marada et al., 2015), and tyrosine kinase inhibitors might cause OATP1B1 inhibition via inhibition of phosphorylation (Sprowl et al., 2016). Endogenous OATP1B biomarkers will be able to demonstrate their clinical relevance in future studies in patients. Whereas the patients generally received drugs other than the potential perpetrator drug to treat their complications, these could have included drugs for which the clinical risk of OATP1Bmediated DDI has not been adequately assessed. In this study, drugs commonly used to treat all patients comprised dexamethasone, diphenhydramine, and famotidine, which at least in vitro, did not inhibit OATP1B1 (Supplemental Fig. 3), but the possibility of OATP1Bmediated DDIs with these drugs needs to be confirmed in further studies.

The changes in endogenous OATP1B biomarkers induced by perpetrator drugs must be extrapolated to the changes in the AUC of OATP1B substrate drugs to suggest the risk of OATP1B-mediated DDI. An important tool for this purpose is the use of a physiologically based pharmacokinetic (PBPK) model. Yoshikado et al. (2008) constructed a PBPK model of CPI that could account for the plasma concentrationtime profiles of CPI after rifampicin administration(Yoshikado et al., 2018). Once an appropriate PBPK model has been constructed for paclitaxel, by analogy with that for CPI, it could be used to help estimate the in vivo $\mathrm{K}_{\mathrm{i}}$ of paclitaxel for OATP1B that would be required to simulate DDIs between paclitaxel and OATP1B substrate drugs, including in cases using other dose regimens for paclitaxel medication. In addition, because the plasma concentrations of CPI and CPIII showed the most rapid decline after the end of the infusion (within 2 hours) of the endogenous substrates tested, PBPK model-based simulation will indicate whether OATP1B1 inhibition by paclitaxel is significant during infusion and for at least 2 hours after the end of infusion, which would enable implementation of an appropriate dosing schedule to avoid significant DDIs.

In conclusion, the present study showed that paclitaxel could inhibit OATP1B1 and OATP1B3 at the dose for the non-small cell lung cancer treatment $\left(200 \mathrm{mg} / \mathrm{m}^{2}\right.$ by a 3 -hour infusion). This study increases the feasibility of transporter-mediated DDI risk assessment in clinical settings.

\section{Authorship Contributions}

Participated in research design: Ishida, Mizuno, Maeda, Sasaki, Fujita, Kusuhara. 
Conducted experiments: Mori, Ishida, Kusumoto, Kondo, Izumi, Nakata, Nozaki, Fujita.

Performed data analysis: Mori, Mizuno, Maeda, Fujita, Kusuhara.

Wrote or contributed to the writing of the manuscript: Mizuno, Fujita, Kusuhara.

\section{References}

Agergaard K, Mau-Sørensen M, Stage TB, Jørgensen TL, Hassel RE, Steffensen KD, Pedersen JW, Milo M, Poulsen SH, Pottegård A, et al. (2017) Clopidogrel-paclitaxel drug-drug interaction: a pharmacoepidemiologic study. Clin Pharmacol Ther 102:547-553.

Akiyama Y, Fujita K, Ishida H, Sunakawa Y, Yamashita K, Kawara K, Miwa K, Saji S, and Sasaki Y (2012) Association of ABCC2 genotype with efficacy of first-line FOLFIRI in Japanese patients with advanced colorectal cancer. Drug Metab Pharmacokinet 27:325-335.

Asaumi R, Menzel K, Lee W, Nunoya K-I, Imawaka H, Hiroyuki K, and Sugiyama Y (2019) Expanded physiologically-based pharmacokinetic model of rifampicin for predicting interactions with drugs and an endogenous biomarker via complex mechanisms including organic anion transporting polypeptide 1B induction. CPT Pharmacometrics Syst Pharmacol DOI: 10.1002/ psp4.12457.

Barnett S, Ogungbenro K, Ménochet K, Shen H, Humphreys WG, and Galetin A (2019) Comprehensive evaluation of the utility of 20 endogenous molecules as biomarkers of OATP1B inhibition compared with rosuvastatin and coproporphyrin I. J Pharmacol Exp Ther 368: 125-135.

Brouwer E, Verweij J, De Bruijn P, Loos WJ, Pillay M, Buijs D, and Sparreboom A (2000) Measurement of fraction unbound paclitaxel in human plasma. Drug Metab Dispos 28: $1141-1145$.

Cheung KWK, Yoshida K, Cheeti S, Chen B, Morley R, Chan IT, Sahasranaman S, and Liu L (2019) GDC-0810 pharmacokinetics and transporter-mediated drug interaction evaluation with an endogenous biomarker in the first-in-human, dose escalation study. Drug Metab Dispos 47 966-973.

Chu X, Liao M, Shen H, Yoshida K, Zur AA, Arya V, Galetin A, Giacomini KM, Hanna I, Kusuhara H, et al.; International Transporter Consortium (2018) Clinical probes and endogenous biomarkers as substrates for transporter drug-drug interaction evaluation: perspectives from the international transporter consortium. Clin Pharmacol Ther 104:836-864.

Deguchi T, Kusuhara H, Takadate A, Endou H, Otagiri M, and Sugiyama Y (2004) Characterization of uremic toxin transport by organic anion transporters in the kidney. Kidney Int $\mathbf{6 5}$ : $162-174$.

Floyd JS, Kaspera R, Marciante KD, Weiss NS, Heckbert SR, Lumley T, Wiggins KL, Tamraz B, Kwok PY, Totah RA, et al. (2012) A screening study of drug-drug interactions in cerivastatin users: an adverse effect of clopidogrel. Clin Pharmacol Ther 91:896-904.

Giacomini KM, Huang SM, Tweedie DJ, Benet LZ, Brouwer KL, Chu X, Dahlin A, Evers R, Fischer V, Hillgren KM, et al.; International Transporter Consortium (2010) Membrane transporters in drug development. Nat Rev Drug Discov 9:215-236.

Hirano M, Maeda K, Shitara Y, and Sugiyama Y (2004) Contribution of OATP2 (OATP1B1) and OATP8 (OATP1B3) to the hepatic uptake of pitavastatin in humans. J Pharmacol Exp Ther 311: 139-146.

Izumi S, Nozaki Y, Maeda K, Komori T, Takenaka O, Kusuhara H, and Sugiyama Y (2015) Investigation of the impact of substrate selection on in vitro organic anion transporting polypeptide 1B1 inhibition profiles for the prediction of drug-drug interactions. Drug Metab Dispos 43:235-247.

King-Ahmad A, Clemens S, Ramanathan R, Zhang Y, Raha N, Zhang Y, Holliman C, Rodrigues $\mathrm{AD}$, and Li F (2018) A fully automated and validated human plasma LC-MS/MS assay for endogenous OATP biomarkers coproporphyrin-I and coproporphyrin-III. Bioanalysis 10: 691-701.

Kunze A, Ediage EN, Dillen L, Monshouwer M, and Snoeys J (2018) Clinical investigation of coproporphyrins as sensitive biomarkers to predict mild to strong OATP1B-mediated drug-drug interactions. Clin Pharmacokinet 57:1559-1570.

Lai Y, Mandlekar S, Shen H, Holenarsipur VK, Langish R, Rajanna P, Murugesan S, Gaud N, Selvam S, Date O, et al. (2016) Coproporphyrins in plasma and urine can Be appropriate clinical biomarkers to recapitulate drug-drug interactions mediated by organic anion transporting polypeptide inhibition. J Pharmacol Exp Ther 358:397-404.

Lee HH and Ho RH (2017) Interindividual and interethnic variability in drug disposition: polymorphisms in organic anion transporting polypeptide 1B1 (OATP1B1; SLCO1B1). Br J Clin Pharmacol 83:1176-1184.

Liu L, Cheeti S, Yoshida K, Choo E, Chen E, Chen B, Gates M, Singel S, Morley R, Ware J, et al. (2018) Effect of OATP1B1/1B3 inhibitor GDC-0810 on the pharmacokinetics of pravastatin and coproporphyrin I/III in healthy female subjects. J Clin Pharmacol 58:1427-1435.

Lowry OH, Rosebrough NJ, Farr AL, and Randall RJ (1951) Protein measurement with the Folin phenol reagent. J Biol Chem 193:265-275.

Maeda K (2015) Organic anion transporting polypeptide (OATP)1B1 and OATP1B3 as important regulators of the pharmacokinetics of substrate drugs. Biol Pharm Bull 38:155-168.

Marada VVVR, Flörl S, Kühne A, Burckhardt G, and Hagos Y (2015) Interaction of human organic anion transporter polypeptides 1B1 and 1B3 with antineoplastic compounds. Eur J Med Chem 92:723-731.

McFeely SJ, Ritchie TK, Yu J, Nordmark A, Levy RH, and Ragueneau-Majlessi I (2019) Identification and evaluation of clinical substrates of organic anion transporting polypeptides 1B1 and 1B3. Clin Transl Sci 12:379-387.
Mizuno T, Hayashi H, and Kusuhara H (2015) Cellular cholesterol accumulation facilitates ubiquitination and lysosomal degradation of cell surface-resident ABCA1. Arterioscler Thromb Vasc Biol 35:1347-1356.

Mori D, Kashihara Y, Yoshikado T, Kimura M, Hirota T, Matsuki S, Maeda K, Irie S, Ieiri I, Sugiyama Y, et al. (2019a) Effect of OATP1B1 genotypes on plasma concentrations of endogenous OATP1B1 substrates and drugs, and their association in healthy volunteers. Drug Metab Pharmacokinet 34:78-86.

Mori D, Kimoto E, Rago B, Kondo Y, King-Ahmad A, Ramanathan R, Wood LS, Johnson JG, Le VH, Vourvahis M, et al. (2019b) Dose-dependent inhibition of OATP1B by rifampicin in healthy volunteers: comprehensive evaluation of candidate biomarkers and OATP1B probe drugs. Clin Pharmacol Ther DOI: 10.1002/cpt.1695 [published ahead of print].

Müller F, Sharma A, König J, and Fromm MF (2018) Biomarkers for in vivo assessment of transporter function. Pharmacol Rev 70:246-277.

Murata H, Ito S, Kusuhara H, Nomura Y, and Taniguchi T (2019) Proposal of a parameter for OATP1B1 inhibition screening at the early drug discovery stage. J Pharm Sci 108:3898-3902 DOI: 10.1016/j.xphs.2019.08.012

Nishizato Y, Ieiri I, Suzuki H, Kimura M, Kawabata K, Hirota T, Takane H, Irie S, Kusuhara H, Urasaki Y, et al. (2003) Polymorphisms of OATP-C (SLC21A6) and OAT3 (SLC22A8) genes: consequences for pravastatin pharmacokinetics. Clin Pharmacol Ther 73:554-565.

Patel M, Taskar KS, and Zamek-Gliszczynski MJ (2016) Importance of hepatic transporters in clinical disposition of drugs and their metabolites. J Clin Pharmacol 56 (Suppl 7):S23-S39.

Rodrigues AD, Taskar KS, Kusuhara H, and Sugiyama Y (2018) Endogenous probes for drug transporters: balancing vision with reality. Clin Pharmacol Ther 103:434-448.

Shen H, Chen W, Drexler DM, Mandlekar S, Holenarsipur VK, Shields EE, Langish R, Sidik K, Gan J, Humphreys WG, et al. (2017) Comparative evaluation of plasma bile acids, dehydroepiandrosterone sulfate, hexadecanedioate, and tetradecanedioate with coproporphyrins I and III as markers of OATP inhibition in healthy subjects. Drug Metab Dispos 45:908-919.

Shitara Y and Sugiyama Y (2017) Preincubation-dependent and long-lasting inhibition of organic anion transporting polypeptide (OATP) and its impact on drug-drug interactions. Pharmacol Ther 177:67-80.

Sonnichsen DS, Liu Q, Schuetz EG, Schuetz JD, Pappo A, and Relling MV (1995) Variability in human cytochrome P450 paclitaxel metabolism. J Pharmacol Exp Ther 275:566-575.

Sprowl JA, Ong SS, Gibson AA, Hu S, Du G, Lin W, Li L, Bharill S, Ness RA, Stecula A, et al. (2016) A phosphotyrosine switch regulates organic cation transporters. Nat Commun 7:10880.

Takehara I, Terashima H, Nakayama T, Yoshikado T, Yoshida M, Furihata K, Watanabe N, Maeda $\mathrm{K}$, Ando O, Sugiyama Y, et al. (2017) Investigation of glycochenodeoxycholate sulfate and chenodeoxycholate glucuronide as surrogate endogenous probes for drug interaction studies of OATP1B1 and OATP1B3 in healthy Japanese volunteers. Pharm Res 34:1601-1614

Takehara I, Watanabe N, Mori D, Ando O, and Kusuhara H (2019) Effect of rifampicin on the plasma concentrations of bile acid-O-sulfates in monkeys and human liver-transplanted chimeric mice with or without bile flow diversion. $J$ Pharm Sci 108:2756-2764.

Takehara I, Yoshikado T, Ishigame K, Mori D, Furihata KI, Watanabe N, Ando O, Maeda K, Sugiyama Y, and Kusuhara H (2018) Comparative study of the dose-dependence of OATP1B inhibition by rifampicin using probe drugs and endogenous substrates in healthy volunteers [published correction appears in Pharm Res (2019) 36:55]. Pharm Res 35:138.

Tátrai P, Schweigler P, Poller B, Domange N, de Wilde R, Hanna I, Gáborik Z, and Huth F (2019) A systematic in vitro investigation of the inhibitor preincubation effect on multiple classes of clinically relevant transporters. Drug Metab Dispos 47:768-778.

Thakare R, Gao H, Kosa RE, Bi Y-A, Varma MVS, Cerny MA, Sharma R, Kuhn M, Huang B, Liu $\mathrm{Y}$, et al. (2017) Leveraging of rifampicin-dosed cynomolgus monkeys to identify bile acid 3-Osulfate conjugates as potential novel biomarkers for organic anion-transporting polypeptides. Drug Metab Dispos 45:721-733.

Vaidyanathan J, Yoshida K, Arya V, and Zhang L (2016) Comparing various in vitro prediction criteria to assess the potential of a new molecular entity to inhibit organic anion transporting polypeptide 1B1. J Clin Pharmacol 56 (Suppl 7):S59-S72.

Yee SW, Brackman DJ, Ennis EA, Sugiyama Y, Kamdem LK, Blanchard R, Galetin A, Zhang L, and Giacomini KM (2018) Influence of transporter polymorphisms on drug disposition and response: a perspective from the international transporter consortium. Clin Pharmacol Ther 104: 803-817.

Yee SW, Giacomini MM, Hsueh CH, Weitz D, Liang X, Goswami S, Kinchen JM, Coelho A, Zur AA, Mertsch K, et al. (2016) Metabolomic and genome-wide association studies reveal potential endogenous biomarkers for OATP1B1. Clin Pharmacol Ther 100:524-536.

Yee SW, Giacomini MM, Shen H, Humphreys WG, Horng H, Brian W, Lai Y, Kroetz DL, and Giacomini KM (2019) Organic anion transporter polypeptide 1B1 polymorphism modulates the extent of drug-drug interaction and associated biomarker levels in healthy volunteers. Clin Transl Sci 12:388-399 DOI: 10.1111/cts.12625.

Yoshikado T, Toshimoto K, Maeda K, Kusuhara H, Kimoto E, Rodrigues AD, Chiba K, and Sugiyama Y (2018) PBPK modeling of coproporphyrin I as an endogenous biomarker for drug interactions involving inhibition of hepatic OATP1B1 and OATP1B3. CPT Pharmacometrics Syst Pharmacol 7:739-747.

Address correspondence to: Dr. Hiroyuki Kusuhara, Laboratory of Molecular Pharmacokinetics, Graduate School of Pharmaceutical Sciences, The University of Tokyo, 7-3-1, Hongo, Bunkyo-ku, Tokyo 113-0033, Japan. E-mail: kusuhara@mol. f.u-tokyo.ac.jp 